\title{
FELINOS DA FLORESTA NACIONAL DE PIRAÍ DO SUL, PARANÁ - BRASIL
}

\section{Elvira DE BASTIANI ${ }^{*}$, Sérgio BAZILIO ${ }^{2}$, KARINA FERreira DE BARROS ${ }^{3}$ \& Gustavo NABRZECKI ${ }^{4}$}

\author{
1,2Universidade Estadual do Paraná (UNESPAR) - Campus União da Vitória. Colegiado de Ciências Biológicas, \\ CEP 84600-000, União da Vitória, PR, Brasil. <elvira_bio@hotmail.com> \\ 3,4Floresta Nacional de Piraí do Sul - Piraí do Sul, PR \\ Recibido: 13/09/2013; aceptado:21/10/2014
}

de Bastiani, E., Bazilio, S., de Barros, K. F. \& Nabrzecki, G. 2015. Felines of Foresta Nacional Piraí do Sul, PR - Brazil. Acta Zoológica Mexicana (n. s.), 31(1): 23-26.

\begin{abstract}
We report the incident of five felines (Leopardus tigrinus, Leopardus wiedii, Leopardus pardalis, Puma concolor and Puma yagouaroundi) through methods not invasive, such as: it looks for straight (visualization) and hint (Photographic traps and collection of material escatológico) in the National Forest of Piraí the South in the state of the Paraná, Brazil.

Key words: non invasive methods, records, Leopardus tigrinus, Leopardus wiedii, Leopardus pardalis, Puma concolor, Puma yagouaroundi.
\end{abstract}

\section{INTRODUÇÃO}

No Brasil ocorrem oito espécies de felinos (Oliveira \& Cassaro 1997): gato-maracajá (Leopardus wiedii), gatodo-mato pequeno ( $L$. trigrinus), gato-do-mato grande ( $L$. geoffroyi), jaguatirica (L. pardalis), gato-palheiro (L. colocolo), jaguarundi (Puma yagouaroundi), onça parda ( $P$. concolor) e onça pintada (Panthera onca).

No Brasil Leopardus wiedii é ocorre em todos os biomas; Leopardus trigrinus com exceção dos Campos Sulinos, habita todos os outros; Leopardus geoffroyi a maioria dos registros provem de áreas florestadas do Rio Grande do Sul, com poucos registros para o Paraná e Mato Grosso do Sul; Leopardus pardalis ocorre em todas as regiões, com exceção do sul do Estado do Rio Grande do Sul; Leopardus colocolo sua distribuição esta incerta, tendo ocorrências registradas nos Estados do Rio Grande do Sul, Paraná, São Paulo, Minas Gerais, Tocantins, oeste da Bahia, Piauí e Maranhão, ocorrendo nos biomas do Cerrado, Pantanal e Campos Sulinos; Puma yagouaroundi habita todos os biomas e estados brasileiros, com exceção o sul do Rio Grande do Sul; Puma concolor ocorre em todos os biomas e estados brasileiros, com exceção o sul do Rio Grande do Sul; Panthera onca ocorre em todos os biomas, exceto nos estados do Rio Grande do Sul, Santa Catarina, Norte de São Paulo, Sergipe, Alagoas, Paraíba, Rio Grande do Norte e Ceará (Reis et al. 2011).

Muitas dessas espécies estão, ao menos potencialmente, ameaçadas de extinção (Chiarello et al. 2008), sendo de Bastiani, E., Bazilio, S., de Barros, K. F. \& Nabrzecki, G. 2015. Felines of Foresta Nacional Piraí do Sul, PR - Brazil. Acta Zoológica Mexicana (n. s.), 31(1): 23-26.

RESUMEN. Se informa la incidencia de cinco felinos (Leopardus tigrinus, Leopardus wiedii, Leopardus pardalis, Puma concolor y Puma yagouaroundi) mediante métodos no invasivos, como son: observación directa (visualización) e indirecta (registro mediante cámarastrampa y recolecciones de material escatológico) en Floresta Nacional (Flona) de Piraí do Sul, estado de Paraná, Brasil.

Palabras clave: métodos no invasivos, registro, Leopardus tigrinus, Leopardus wiedii, Leopardus pardalis, Puma concolor, Puma yagouaroundi.

as principais causas a redução, fragmentação de habitats (Woodroffe \& Ginsberg 1998; Freitas et al. 2010; Martin et al. 2012) e pressão de caça (Reis et al. 2011). Tanto em nível estadual quando nacional L. tigrinus, $L$. pardalis, $P$. concolor, L. wiedii encontram-se "vulnerável” (Mikich \& Bérnelis 2004; Chiarello et al. 2008). E em nível global L. tigrinus encontra-se "vulnerável" e $L$. wiedii "quase ameaçado" (IUCN 2014). Para a regiões dos Campos Gerais no estado do Paraná são escassos estudos publicados abordando ocorrência de felinos, a maioria dos registros é relatada em estudos sobre a dieta (Vidolin 2004; RochaMendes 2005; Quadros \& Monteiro-Filho 2006a; 2006b; Azevedo 2008; Rocha-Mendes et al. 2010; Silva-Pereira et al. 2011;) e em levantamentos (Tomás \& Miranda 2003; Peters et al. 2010) de mamíferos de médio e grande porte. A presente nota amplia a distribuição geográfica de Leopardus tigrinus, Leopardus wiedii, Leopardus pardalis, Puma concolor e Puma yagouaroundi com base em dados concretos de busca de vestígios diretos e indiretos e de armadilhamento fotográfico na Floresta Nacional de Piraí do Sul no estado do Paraná.

\section{MATERIAL E MÉTODOS}

A Floresta Nacional (Flona) de Piraí do Sul (24³4'21.6' 'S; 4955'33.6' 'Walt. 1085 m) localiza-se no município de Piraí do Sul, Paraná (Figura 1). A Flona possui uma área de 150 hectares, dos quais sete constituem em reflorestamentos de Araucária, 0.5 de Imbuia, 39 com Pinus sp. 


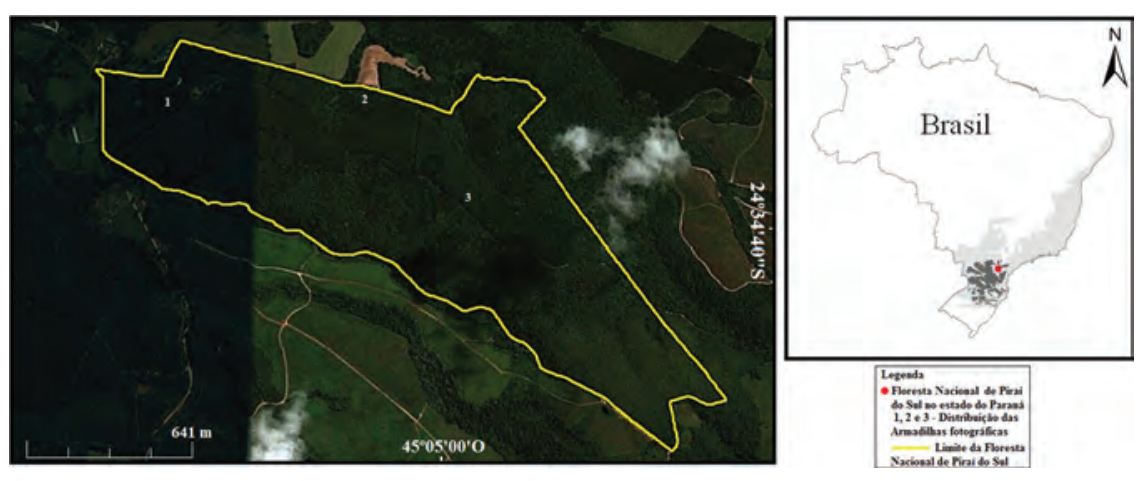

Figura 1. Mapa da estrutura da Floresta Nacional de Piraí do Sul Fonte: Flona de Piraí do Sul.

e o restante da área é composto por florestas nativas em diferentes estágios sucessionais (Fig. 1). A área da flona apresenta um relevo suavemente ondulado, com pequena elevação na parte sudeste, na Serra das Pedras, onde se encontram as nascentes dando origem aos cursos d'água. O clima segundo a classificação de Köppen é do tipo $C f b$, com verões moderadamente quentes e incidência de geadas no inverno.

A coleta de dados ocorreu mensalmente com duração de três dias consecutivos, entre março de 2012 a março de 2013. Para a coleta dos dados foram aplicadas três metodologias não invasivas distintas: busca de vestígios diretos e indiretos, armadilhamento fotográfico (Karanth et al. 2004; Sanderson \& Trolle 2005) e análise das fezes coletadas (Tomás \& Miranda 2003; Quadros \& Monteiro-Filho 2006a; 2006b; 2011; Silva-Pereira et al., 2011). Foram instaladas três armadilhas fotográficas (Modelo Bushnell), sem iscas, as quais permaneceram ligadas 24 horas por dia, totalizando 21.600 horas em que as armadilhas estiveram ativas. Todas as amostras fecais foram georreferenciadas (Garmim-Etrex) e fotografadas. Para a análise e identificação das fezes, seguiram-se os procedimentos de Quadros e Monteiro-Filho (2006a) e após comparou-se com o laminário de referência dos autores.

\section{RESULTADOS}

Com esforço de $280 \mathrm{~km}$ a percorridos a pé, totalizando 135 horas de esforço amostral de busca direta, dentre as quais foram coletadas 43 amostras fecais, 27 registros de pegadas e 21.600 horas de armadilhamento fotográfico, obtendo 1.844 fotos das quais 36 são pertencem a Família Felidae. Foram registradas cinco espécies de felinos na Flona de Piraí do Sul sendo: Leopardus pardalis, Leopardus tigrinus, Leopardus wiedii, Puma concolor (Fig. 2), Puma yagouaroundi. Através da análise do material escatológico foi encontrado o pelo do autor, ou seja, da espécie que defecou e através da identificação dos padrões micro- estruturais dos pelos foram identificados de acordo com Quadros e Monteiro-Filho (2006a) cinco espécies por este método, constituindo em 53\% das amostras de L. pardalis, $19 \%$ de $P$. yagouaroundi, $12 \%$ de L. tigrinus, $9 \%$ de $P$. concolor e $7 \%$ de $L$. wiedii. Através de pegadas $69 \%$ dos registros foram de $P$. concolor, $23 \%$ de $L$. pardalis e $8 \%$ de $L$. tigrinus, não sendo registradas $L$. wiedii e $P$. yagouaroundi por este método. Por meio de visualização foi registrado na região sudeste da flona uma fêmea de jaguatirica com seu filhote. E através do armadilhamento fotográfico a espécie mais abundante foi $P$. concolor com $69 \%$ dos registros fotográficos (dentre estas, nove foram com seus dois filhotes), seguida de L pardalis com $13 \%$ e $L$. tigrinus, $L$. wiedii e P. yagouaroundi com $6 \%$ para cada espécie.

Das espécies de felinos encontradas na área da Flona de Piraí do Sul no Paraná a maioria encontra-se "vulnerável” ou "quase ameaçada”, com exceção do gato-mourisco ( $P$. yaguaroundi), que apresenta dados insuficientes para classificação de seu status segundo Mikich e Bérnils (2004). A baixa detecção de algumas espécies pode ser influenciada pela presença de uma espécie dominante, pois a competição por recursos pode afetar a dinâmica populacional do outro, ocorendo que de fato uma sobreposição na dieta entre os pequenos felídeos neotropicais Oliveira et al. (2010). Oliveira-Santos et al. (2012) ressalta que registros recentes de $L$. tigrinus em paisagens degradadas de Mata Atlântica do Brasil, evidenciam que os mesmos podem prosperar mesmo em ambientes agressivos onde outros gatos já foram extintos, podendo assumir um papel de predador, na ausência dos felinos de maior porte nas comunidades. Os resultados encontrados na flona corroboram com os estudos de Vidolin \& Góss Braga (2004) e com Moro (2007) com exceção da onça pintada que não foi registrada na flona e que provavelmente esteja extinta na região de estudo.

Das sete espécies de felinos citadas para o estado do Paraná (Mikich e Bérnils 2004), cinco ocorrem na Flona 

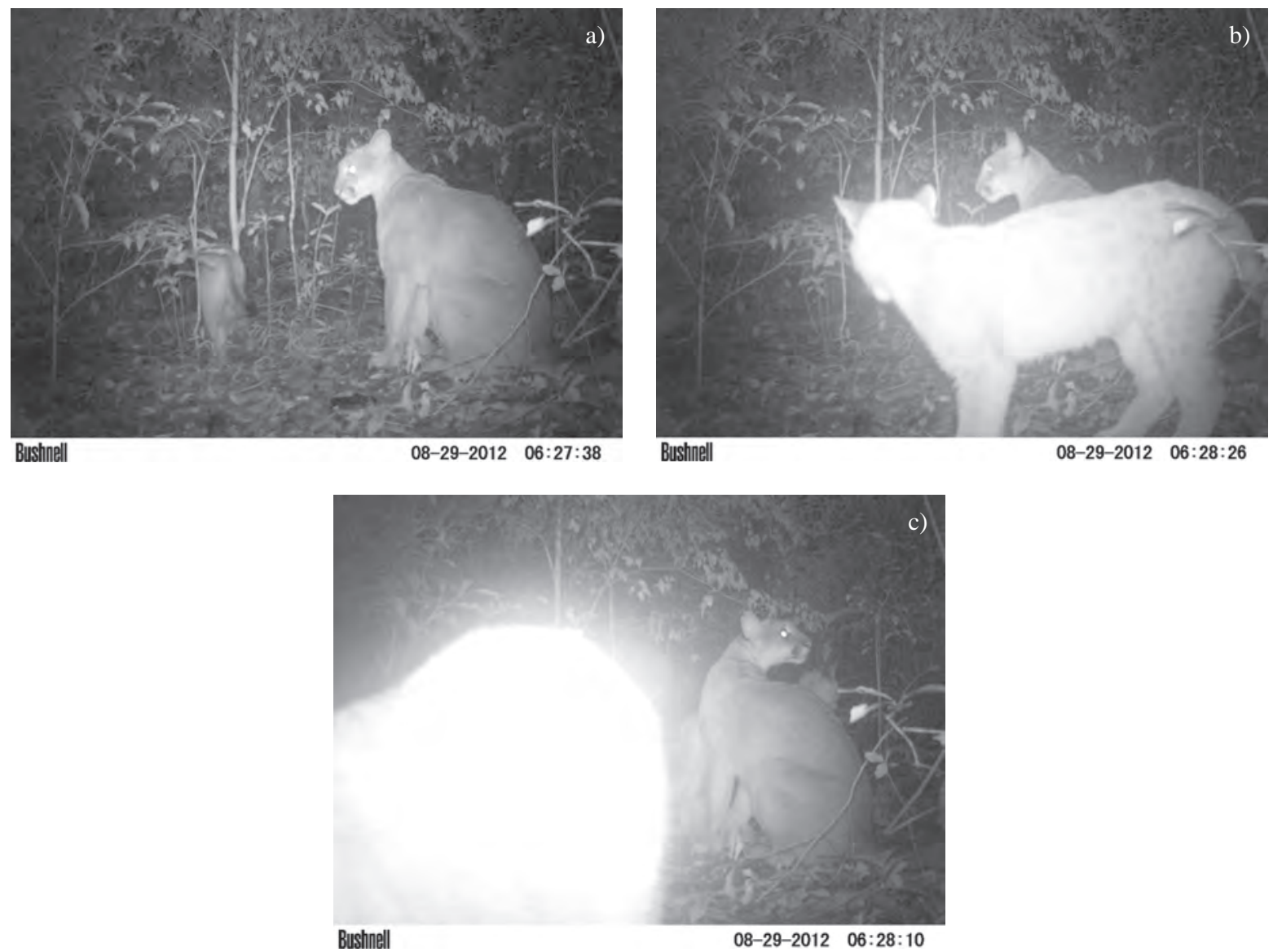

Figura 2. a,b e c - Registro fotográfico de Puma concolor na Floresta Nacional de Piraí do Sul no estado do Paraná, Brasil.

de Piraí do Sul. A presença destas espécies levanta a possibilidade da criação de um corredor biológico, pois área de estudo corresponde a uma importante região, fazendo conexão entre as áreas florestadas da empresa Iguaçu S/A e pequenas fazendas com gado e agricultura no entorno. Estudos direcionados para distribuição dos felinos são poucos, por isso considera-se necessário visto que espécies anteriormente registradas em áreas próximas por Vidolin \& Góss Braga (2004) e Moro (2007) como Panthera onca não foi registrada neste estudo. Manter suas populações é extremamente importante, especialmente aqueles que estão sob ameaça de extinção, reforçando a necessidade premente de ações e estratégias em prol de sua conservação, podendo estes dados servir como base para elaboração de políticas públicas visando à proteção da biodiversidade da mastofauna ameaçada no Plano de manejo Da Unidade.

\section{REFERÊNCIAS}

Azevedo, C. C. F. 2008. Food habits and livestock depredation of sympatric jaguars and puma the Iguaçu National Park Area, South Brazil. Biotropica, 40: 494-500.
Chiarello, A. G., Aguiar, L. M. S., Cerqueira, R., Melo, F. R., Rodrigues, F. H. G. \& Silva, V. M. F. 2008. Mamíferos, pp. 689874. In: A. B. M. Machado, G. M. Drummond \& A. P. Paglia. Livro Vermelho da fauna brasileira ameaçada de extinção. Belo Horizonte, MG. Fundação Biodiversitas.

Freitas, S. R. , Hawbaker, T. J. \& Metzger, J. P. 2010. Effects of roads, topography, and land use on forest cover dynamics in the Brazilian Atlantic Forest. Forest Ecology and Management, 259: 410-417.

Karanth, K. U., Nichols, J. D. \& Kumar, N. S. 2004. Photographic sampling of elusive mammals in tropical forests, pp. 229-247. In: Thompson WL. (Ed.) Sampling rare or elusive species: concepts and techniques for estimating population parameters. Island Press, Washington, DC.

Martin, P. S., Gheler-Costa, C., Lopes, P. C., Rosalino, L. M. \& Verdade, L. M. 2012. Terrestrial non-volant small mammals in agro-silvicultural landscapes of Southeastern Brazil. Forest Ecology and Management, 282: 185-195.

Mikich, S. B., \& Bérnils, R. S. 2004. Livro Vermelho da Fauna Ameaçada no Estado do Paraná. Curitiba: Instituto Ambiental do Paraná.

Moro, R. S. 2007. Patrimônio natural dos campos gerais do Paraná, In: F. Góss Braga. Capítulo 12 Mamíferos dos Campos Gerais $\left(1^{\circ}\right.$ Eds). Brasil. Ponta Grossa, UEPG, pp. 123-133.

Oliveira, T. G. \& Cassaro, K. 1997. Guia de identificação dos felinos brasileiros. Sociedade de Zoológicos do Brasil. Fundação parque Zoológico de São Paulo, 60 pp. 
Oliveira, T. G., Tortato, M. A., Silveira, L., Kasper, C. B., Mazim, F. D., Lucherini. M., Jácomo, A. T., Bonifacio, J., Soares, G., Marques, R. V. \& Sunquist, M. 2010. Ocelot ecology and its effect on the small-felid guild in the lowland neotropics, pp. 559580. In: Macdoweld, D. W. \& A. J. Loveridge. (Eds.). Biology and conservation of wild cats. Oxford University Press.

Oliveira-Santos, L. G. R., Graipel, M. E., Tortato, M. A., Zucco, C. A., Cáceres, N. C. \& Goulart, F. V. 2012. Abundance changes and activity flexibility of the oncilla, Leopardus tigrinus (Carnivora: Felidae), appear to reflect avoidance of conflict. Zoologia (Curitiba), 29: 115-120.

Peters, F. B., Roth, P. R. O., Machado, L. F., Coelho, E. L., Jung, D. M. H. \& Christoff, A. U. 2010. Assembléia de mamíferos dos agroecossistemas constituintes da bacia hidrográfica do rio da Várzea, Rio Grande do Sul. Biotemas, 4: 91-107.

Quadros, J. \& Monteiro-Filho, E. L. A. 2006a. Coleta e preparação de pêlos de mamíferos para identificação em microscopia óptica. Revista Brasileira de Zoologia, 1: 274-278.

Quadros, J. \& Monteiro-Filho, E. L. A. 2006b. Revisão conceitual, padrões microestruturais e proposta nomenclatória para os pêlosguarda de mamíferos brasileiros. Revista Brasileira de Zoologia, 23: 279-292.

Reis, N. R., Peracchi, A. L., Pedro, W. A. \& Lima, I. P. 2011. Mamíferos do Brasil. pp. 439. In: Cheida, C. C., Nalkano-Oliveira, E., Fusco-Costa, R., Rocha-Mendes, F. \& Quadros, J. Ordem carnívora. Londrina: Universidade de Londrina.

Rocha-Mende, F., Bos Mikich, S., Quadros, J., Pedro, W. A. 2010. Feeding ecology of carnivores (Mammalia, Carnivora) in Atlantic Forest remmants, Southern Brazil, Biota Neotropica, 104: 1-10.
Rocha-Mendes, F. 2005. Ecologia alimentar de carnívoros (Mammalia: Carnívora) e elementos de Etnozoologia do município de Fênix, Paraná, Brasil. Dissertação de Mestrado. Universidade Estadual Paulista. São José do Rio Preto.

Sanderson, J. G. \& Trolle, M. 2005. Monitoring elusive mammals unattended. Cameras reveal secrets of some of the world's wildest places. American Scientist, 93: 148-155

Silva-Pereira, J., Moro-Rios, F. R., Bilski, D. \& Passos, F. C. 2011. Diets of three sympatric Neotropical small cats: Food niche overlap and interspecies differences in prey consumption. Mammalian Biology, 76: 308-312.

The IUCN Red List of Threatened Species. Version 2014.2. <www. iucnredlist.org>. Acesso em: 15 Outubro 2014.

Tomás, W. M. \& Miranda, G. H. B. 2003. Uso de armadilhas fotográficas em levantamentos populacionais. In: Cullen, J. R., Rudran, L. R. \& Valladares-Pádua, C. (Eds.) Métodos de estudos em Biologia da Conservação e Manejo da Vida Silvestre. pp. 243265.

Vidolin, G. P. \& Góss Braga, F. 2004. Ocorrência e uso da área por carnívoros silvestres no Parque Estadual do Cerrado, Jaguariaíva, Paraná. Caderno Biodiversidade, 4: 29-36.

Vidolin, G. P. 2004. Aspectos bio-ecológicos de Puma concolor (Linnaeus, 1771), Leopardus pardalis (Linnaeus, 1758) e Leopardus tigrinus (Schreber, 1775) na Reserva Natural Salto Morato, Guaraqueçaba, Paraná, Brasil. Dissertação de Mestrado. Universidade Federal do Paraná. Curitiba.

Woodroffe, R. \& Ginsberg, J. R. 1998. Edge effects and the extinction of populations inside protected areas. Science 280: 21262128. 